\title{
Endoscopic ultrasound (EUS) aspect of duodenal bulb invasion by multifocal malignant pancreatic intraductal papillary mucinous neoplasm (IPMN)
}

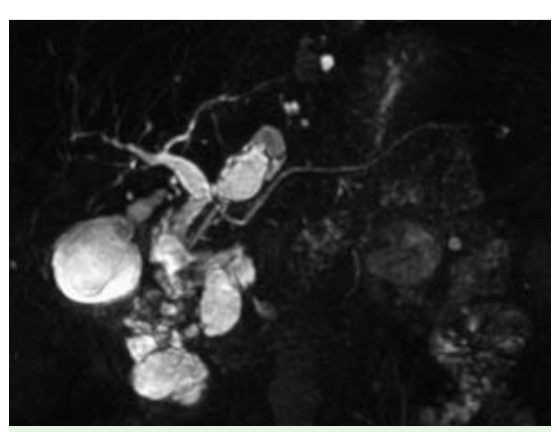

Fig. 1 Magnetic resonance cholangiopancreatography shows multiple cysts.

An 83-year-old woman came to our institute with abdominal pain, tomographic evidence of multiple pancreatic cystic lesions, and abnormal thickening of the pyloroduodenal region. Magnetic resonance imaging showed multiple cysts in the pancreatic body, isthmus, head, and uncinate process, some with clear communication with the pancreatic duct ( $\bullet$ Fig. 1), and a parietal thickening of the duodenum with contrast enhancement, in very close proximity to a cystic lesion in the pancreatic isthmus ( Fig. 2a). Endoscopic ultrasound (EUS) showed a multiloculated, septated cystic lesion in the pancreatic isthmus, with a hypoechoic pericystic lesion invading the duodenal bulb ( $\bullet$ Fig. 2 b). Gastroscopy confirmed a villous lesion in the duodenal bulb, involving the inferoposterior wall ( $\nabla$ Fig. 2c). EUS showed that the intraduodenal lesion was heterogeneous with a solid-cystic aspect ( $\bullet$ Fig. 2d). Biopsy of the duodenal lesion confirmed duodenal wall invasion by a malignant intraductal papillary mucinous neoplasm (IPMN) ( $\bullet$ Fig. 2 e,f).

EUS fine-needle aspiration cytology of a pancreatic cyst in the uncinate process showed atypical cells with severe dysplasia, strongly suggestive of malignant IPMN ( $\bullet$ Fig. 3), as previously reported [1]. This report highlights a case of a degenerating multifocal IPMN invading the duodenal bulb. Benign and malignant IPMNs can extend to surrounding organs (duodenum, common bile duct, stomach) [2-5]. In benign lesions, mucus produced by tumor cells is compressed and exerts pressure, inducing ischemia, atrophy, and disappearance of the gastroduodenal or
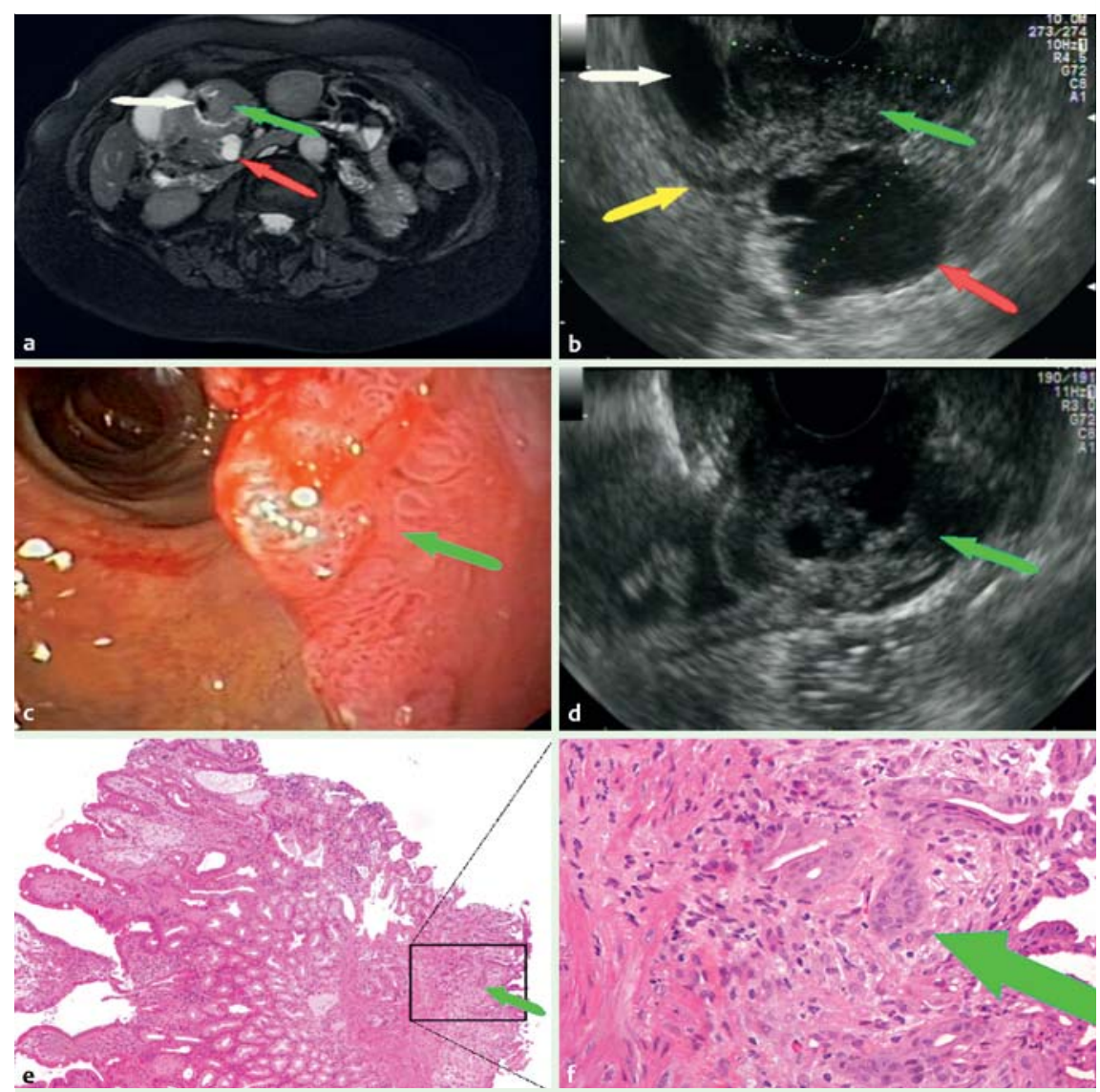

Fig. 2 a Magnetic resonance imaging (MRI) showing the cystic lesion in the pancreatic isthmus, and parietal thickening of the duodenal bulb wall (white arrow, duodenal lumen; red arrow, pancreatic isthmus cyst; green arrow, intraduodenal lesion). b Endoscopic ultrasound (EUS) aspect of the cystic lesion in the pancreatic isthmus. A hypoechoic pericystic lesion invades the duodenal bulb wall, and there is interruption of the muscularis propria (white arrow, duodenal lumen; yellow arrow, muscularis propria of the duodenal bulb wall; red arrow, pancreatic isthmus cyst; green arrow, intraduodenal lesion). c Duodenoscopic image of villous lesion in the duodenal bulb (green arrow, intraduodenal lesion). d EUS aspect of duodenal bulb lesion as a hypoechoic solid-cystic mass. (Green arrow, intraduodenal lesion.) e Duodenal bulb lesion biopsy. Histological examination shows submucosal invasion with incipient interruption of muscularis mucosae by adenocarcinoma. Note the intact mucosa layer (green arrow, intraduodenal lesion). $\mathbf{f}$ Box magnification showing adenocarcinomatous component of malignant intraductal papillary mucinous neoplasm (IPMN) (green arrow, intraduodenal lesion).

bile duct wall, with final penetration by the IPMN; these lesions are called the "automatic type." On the other hand, malignant lesions can directly infiltrate surrounding organs and are called the "invasive type" [2-3].

The intraluminal lesion of an invading IPMN can assume, at EUS, the same solidcystic pattern as the malignant pancreatic lesion. It is also worth bearing in mind that there can be synchronous multifocal degeneration of IPMNs, as evidenced in our case by the presence of a malignant pancreatic isthmus IPMN invading the duodenal bulb, and a cytology-proven malignant IPMN in the uncinate process. Both these aspects should be taken into consideration for diagnostic purposes and therapeutic strategies.

Endoscopy_UCTN_Code_CCL_1AZ_2AB

Competing interests: None 


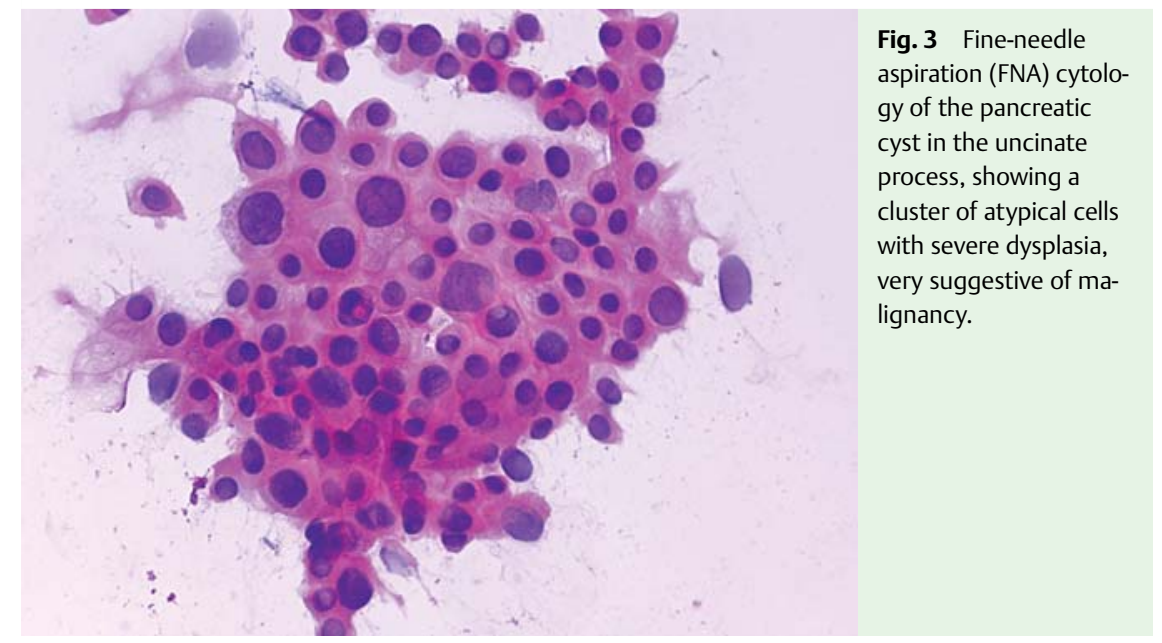

L. Barresi ${ }^{1}$, I. Tarantino ${ }^{1}$, G. Curcio ${ }^{1}$, A. Granata', R. Liotta ${ }^{2}$, R. Gentile ${ }^{2}$, M. Traina ${ }^{1}$

${ }^{1}$ Gastroenterology and Endoscopy Unit, Mediterranean Institute for Transplantation and Advanced Specialized Therapies (IsMeTT), Palermo, Italy

2 Pathology Service, Mediterranean Institute for Transplantation and Advanced Specialized Therapies (IsMeTT), Palermo, Italy
4 Jausset F, Delvaux M, Dumitriu D et al. Benign intraductal papillary-mucinous neoplasm of the pancreas associated with spontaneous pancreaticogastric and pancreaticoduodenal fistulas. Digestion 2010; 82: $42-46$

5 Jung IS, Shim C, Cheon S et al. Invasive intraductal papillary mucinous tumor of the pancreas with simultaneous invasion of the stomach and duodenum. Endoscopy 2004; 36: $186-189$

\section{Bibliography}

DOI $10.1055 / \mathrm{s}-0030-1256617$

Endoscopy 2011; 43: E270 -E271

(c) Georg Thieme Verlag KG Stuttgart · New York . ISSN 0013-726X

\section{Corresponding author}

\section{Barresi, MD}

Gastroenterology and Endoscopy Unit Mediterranean Institute for Transplantation and Advanced Specialized Therapies (IsMeTT)

\section{Via Tricomi 1}

Palermo 90127

Italy

Fax: +39-91-2192400

Ibarresi@ismett.edu 